\title{
Estudio preliminar de la fauna en el morro de basuras de Moravia y presencia de metales pesados en artrópodos y roedores
}

\author{
María Solange Sánchez $^{1 *}$, Alejandra Bedoya ${ }^{2}$, Rolando Barahona $^{3}$ \\ ${ }^{1}$ Facultad de Ciencias, Pontificia Universidad Javeriana, Bogotá, D.C., Colombia \\ ${ }^{2}$ Investigadora independiente. Colombia \\ ${ }^{3}$ Departamento de Producción Animal, Facultad de Ciencias Agropecuarias, Universidad Nacional de Colombia, \\ Medellín Campus. Medellín, Colombia \\ *sanchez.mariasolange@gmail.com
}

Recibido: 08-03-2010; Aceptado: 25-04-2010

\begin{abstract}
Resumen
La caracterización de las especies presentes en un sitio determinado a través de inventarios biológicos, brinda información sobre su diversidad, incluso cuando es realizado en ambientes intervenidos como los basureros a cielo abierto, lugares considerados como una amenaza para la salud, dado que allí suelen acumularse metales pesados, reconocidos como riesgo para la salud y el ambiente. Objetivos. Realizar la caracterización preliminar de artrópodos y pequeños mamíferos no voladores del morro de basuras de Moravia, así como la determinación de metales pesados en muestras de tejido animal. Materiales y Métodos. Para la captura de artrópodos se utilizaron trampas de caída y captura manual. Los roedores se capturaron con trampas Sherman. El análisis de metales pesados en tejido animal se realizó mediante espectroscopia atómica. Resultados. Se identificaron 9 órdenes, 51 familias y 75 morfoespecies de insectos, 5 familias de arañas, dos especies de sírfidos y una especie de milpiés; Mus musculus fue la única especie de roedor identificado. El análisis de metales pesados mostró diversas concentraciones de $\mathrm{Pb}$ y $\mathrm{Cd}$ principalmente. El contenido máximo de $\mathrm{Pb}(45,05 \mathrm{mg} / \mathrm{kg})$ se encontró en muestras de los especímenes de M. musculus y el contenido más alto de Cd $(10,31 \mathrm{mg} / \mathrm{kg})$ se encontró en las muestras de arañas de la familia Gasteracantha. Conclusiones. El morro de Moravia presenta condiciones que permiten el establecimiento de una comunidad de artrópodos con diversas dietas y nichos. Asimismo, se corrobora la transferencia de metales pesados desde la matriz de residuos hacia niveles superiores de la cadena trófica.
\end{abstract}

Palabras clave: artrópodos, cadmio, inventario biológico, Mus musculus, plomo.

\begin{abstract}
Preliminary study of the fauna in the Morro de Moravia open dump and presence of heavy metals in arthropods and rodents. Characterization of the species present in a given locality by means of biological inventories provides information about diversity, even if it is done in disturbed environments such as open dumps, which are considered a threat for health due to the accumulation of heavy metals that are well known for their negative effects on human health and the environment. Objectives. To carry out a preliminary characterization of arthropods and non-flying small mammals in the Morro de Moravia open dump, and to determine the heavy metal contents in animal tissue samples. Materials and Methods. Arthropods were collected with pitfall traps and manually. Rodents were captured with Sherman traps. The heavy metal analysis was carried out with atomic spectroscopy. Results. A total of 9 orders, 51 families and 75 morphospecies of insects, 5 families of spiders, two syrphid and a millipede species were identified; Mus musculus was the only small mammal species identified. Heavy metal analysis showed various concentrations mainly of $\mathrm{Pb}$ and $\mathrm{Cd}$. The highest content of $\mathrm{Pb}(45.05 \mathrm{mg} / \mathrm{kg}) \mathrm{was}$ found in samples of M. musculus and the highest Cd content $(10.31 \mathrm{mg} / \mathrm{kg}$ ) was found in spiders of the family Gasteracantha. Conclusions. The Morro de Moravia open dump provides conditions that allow the establishment of an arthropod community with various diets and niches. The transference of heavy metals from the residue matrix towards higher levels of the trophic chain was corroborated.
\end{abstract}

Key words: arthropods, biological inventories, cadmium, Mus musculus, lead 


\section{Resumo}

Estudo preliminar da fauna no monte de lixo da Moravia e presença de metais pesados em artrópodes e roedores A caracterização das espécies presentes num determinado local através de inventários biológicos, fornece informação sobre sua diversidade, mesmo quando é realizada em ambientes como os lixos a céu aberto, locais considerados como uma ameaça para a saúde, devido a que freqüentemente acumulam metais pesados, reconhecidos como um risco para a saúde e o meio ambiente. Objetivos. Realizar a caracterização preliminar de artrópodes e pequenos mamíferos não-voadores do monte de lixo da Moravia, e a determinação de metais pesados em amostras de tecido animal. Materiais e Métodos. Para a captura dos artrópodes utilizaram-se armadilhas de queda e captura manual. Os roedores foram capturados com armadilhas Sherman. A análise de metais pesados nos tecidos dos animais foi realizada por espectroscopia atômica. Resultados. Foram identificados 9 ordens, 51 famílias e 75 morfoespécies de insetos, 5 famílias de aranhas, duas espécies de moscas sírfidos e uma de centopéia, Mus musculus foi a única espécie de roedor encontrado. A análise de metais pesados a presenteou diferentes concentrações de $\mathrm{Pb}$ e Cd principalmente. O teor máximo de $\mathrm{Pb}(45,05 \mathrm{mg} / \mathrm{kg})$ foi encontrado em amostras de espécimes de $M$. musculus e os maiores teores de $\mathrm{Cd}(10,31 \mathrm{mg} / \mathrm{kg})$ foi encontrado nas amostras de aranhas da família Gasteracantha. Conclusões. O monte de lixo da Moravia apresenta condições que permitem o estabelecimento de uma comunidade de artrópodes com dietas diferentes e nichos. Também, se confirma à transferência de metais pesados a partir da matriz de resíduos para níveis mais altos da cadeia alimentar.

Palavras-chave: artrópodes, cádmio, inventário biológico, Mus musculus, chumbo

\section{Introducción}

La caracterización de las especies presentes en un ambiente determinado refleja la variedad de formas de vida. Dicha caracterización se logra mediante la realización de inventarios biológicos que suministran una lista de taxones presentes en dicho lugar. Sin embargo, al inventariar y caracterizar el estado de la biodiversidad de un sitio, es indispensable restringir los muestreos a sólo unos componentes de la biodiversidad, ya que el conocimiento taxonómico, el financiamiento y el esfuerzo necesario para obtener información, son algunos de los limitantes para la ejecución de este tipo de estudios (1).

Dentro del inventario de fauna, el grupo de los artrópodos y el de pequeños mamíferos no voladores han sido considerados como bioindicadores de diversos parámetros, entre ellos el grado de polución o de intervención antropogénica. Los artrópodos se caracterizan por su permanencia histórica en el planeta, su alta diversidad y su adaptación a diversos ambientes (2). Por otra parte, dentro del grupo de pequeños mamíferos no voladores el orden Rodentia representa casi un tercio de todos los mamíferos y en él se encuentran algunas de las plagas que padece el hombre como son el ratón casero (Mus musculus), la rata común (Rattus norvegicus) y la rata negra (Rattus rattus), las cuales de la mano del hombre, se han hecho ecuménicas. Debido a su abundancia, amplia distribución, patrones de migración en corta distancia, hábitos alimenticios, alta tasa de reproducción y facilidad de captura, son considerados bioindicadores adecuados en sitios intervenidos (3).

Los basureros y rellenos sanitarios, lugares en que se lleva a cabo disposición final de los residuos producidos por una comunidad, se caracterizan por su riqueza en recursos alimenticios altos en energía, lo que los hace un sitio atrac- tivo para hospedar gran variedad de organismos, así como por su grado de contaminación por diversas sustancias contaminantes. Entre dichos contaminantes se encuentran los metales pesados, que se caracterizan por presentar una densidad mayor a $6 \mathrm{~g} / \mathrm{cm} 3$, su condición de no degradabilidad a través de procesos biológicos, algunos de ellos no tienen función biológica conocida y la acumulación en diferentes componentes de la red alimenticia, razones por las cuales se han calificado como una grave amenaza para la salud (4). La exposición a metales pesados se ha asociado con graves problemas de salud en la población humana que van desde dermatitis y reacciones alérgicas, hasta daños neurológicos y cáncer (5 - 7).

El basurero a cielo abierto conocido como el Morro de Moravia se desginó como el sitio de disposición final de los residuos de la ciudad de Medellín y operó durante 1970 a 1984. Como consecuencia de dicha disposición, el área se transformó en un cerro de 7,6 has y $30 \mathrm{~m}$ de altura. Durante la operación del basurero y después de su clausura, el cerro ha sido poblado continuamente y en la actualidad viven allí alrededor de 4000 personas. La situación descrita ha sido considerada como un problema de salud pública y una amenza para el entorno. En vista de lo anterior, la entidad oficial Area Metropolitana del Valle de Aburrá ha iniciado estudios para determinar el grado de contaminación en el cerro y ha dispuesto medidas de reducción del riesgo para la población. Entre estas últimas, se encuentra la reubicación de la población residente en el cerro. Por tal motivo, en algunos lotes del cerro (donde anteriormente se encontraban las viviendas), se ha establecido un proceso de revegetación, en su mayoría de ocurrencia espontánea. De acuerdo a los resultados obtenidos en el estudio de la zona (8), la matriz de residuos (MR) contiene altas concentraciones de metales pesados $(\mathrm{Hg}$, $\mathrm{Pb}, \mathrm{Cr}, \mathrm{Cd}$ y $\mathrm{Ni}$ ), excediendo por una amplio rango los 
límites permitidos en las normatividades Colombiana (9) y Europea (10). En el mismo estudio se evidenció que las plantas colonizadoras del cerro absorbían en diverso grado los metales pesados analizados, sugiriendo la transferencia de los mismos desde la matriz de residuos hacia los organismos vivos en Moravia.

Con el presente estudio se pretende realizar una descripción de la composición de las comunidades de artrópodos y pequeños mamíferos no voladores (roedores) presentes en el Morro de basuras de Moravia, así como determinar si existe transferencia de metales pesados hacia niveles consumidores en la cadena trófica en el cerro de basuras de Moravia. Para esto se realizó una caracterización de estos grupos faunísticos en el antiguo botadero y se determinó el nivel de contaminación por metales pesados $(\mathrm{Pb}, \mathrm{Cr}, \mathrm{Cd}$ y Ni) en sus tejidos.

\section{Materiales y métodos}

Para la caracterización de la fauna presente en el cerro de basuras de Moravia, se seleccionaron grupos faunísticos adaptados a un amplio rango de ambientes, tal como es el caso de los artrópodos (invertebrados) y los roedores (del grupo de los pequeños mamíferos no voladores).

\section{Muestreo de Insectos}

Para el muestreo de insectos se establecieron tres estaciones de muestreo, las cuales consistieron en tres trampas de caída pitfall, generalmente utilizadas en el monitoreo de este grupo faunístico (11). Las trampas tenían diferentes tipos de atrayente (coprocebo, fruta y pescado en descomposición) y estaban separadas una de otra por una distancia mínima de $30 \mathrm{~m}$. Cada trampa de caída consistió de un vaso plástico de 16 onzas enterrado a ras de la superficie, suspendido con un alambre sobre el vaso. En cada trampa se colocó una copa plástica de 2,5 onzas con el atrayente correspondiente. Para conservar los insectos capturados, se utilizó etanol al 70\% (v/v) (1). Estas trampas permanecieron en el campo 48 horas sin ser recebadas. Adicionalmente, se realizó un muestreo mediante red entomológica o jama en una parcela de 2 x 2 m dentro de la cual se realizó captura de los insectos atrapados allí presentes (1).

Todo el material biológico colectado se trasladó en bolsas plásticas rotuladas al Laboratorio de Entomología de la Universidad Nacional de Colombia, Sede Medellín. La identificación (familia, género y morfoespecie), se llevó a cabo mediante el uso de claves taxonómicas y por medio de la comparación de los ejemplares con los individuos del Museo Entomológico Francisco Luís Gallego (MEFLG).

\section{Muestreo de arañas}

Para el muestreo de arañas se establecieron tres estaciones, muestreando en cada una de ellas un área de $1 \mathrm{~m}^{2}$. En cada estación se realizó una inspección visual y las telas de araña identificadas se espolvorearon con fécula de maíz para mejorar la visibilidad de la tela y facilitar la captura de las arañas presentes. Se capturaron aquellas que se encontraban en el área mediante captura directa o búsqueda activa de especímenes, según la metodología de Turnbull (12).

\section{Muestreo de pequeños mamíferos no voladores}

Para el muestreo de roedores, se utilizaron 15 trampas tipo Sherman, que se ubicaron al interior de viviendas de algunos pobladores del cerro. Este tipo de trampa (live trap) se utiliza generalmente para capturar especímenes sin causar su muerte (13).

Cada trampa contenía un pedazo de carne como atrayente y se dejó en el sitio de muestreo por un espacio de 24 horas. Los individuos capturados se transportaron al laboratorio de Nutrición Animal de la Universidad Nacional de Colombia, sede Medellín y para su traslado se utilizaron bolsas de tela y guantes de carnaza. Una vez en el laboratorio, los especímenes se clasificaron por medio de comparaciones morfológicas con claves de identificación.

\section{Determinación de metales pesados en tejido animal}

Los especímenes de insectos y arañas capturados se conservaron en etanol (solución acuosa al 70\% (v/v)) y se refrigeraron a $4^{\circ} \mathrm{C}$ hasta el momento de la determinación de $\mathrm{Pb}, \mathrm{Cr}, \mathrm{Cd}$ y Ni. Para el análisis de metales pesados, la muestra consistió de todos los especímenes (cuerpo completo) capturados y pertenecientes a la misma familia, esto con el fin de obtener una cantidad suficiente de muestra. Los especímenes de roedores capturados e identificados, se sacrificaron por medio de inhalación con éter. Inmediatamente se procedió a realizar la necropsia y separación de algunos órganos y de esta manera obtener diferentes muestras para análisis de metales. Estas muestras estuvieron 
conformadas por el hígado, los riñones, la cabeza y el resto del cuerpo de los roedores. Dicha separación se realizó de acuerdo a los reportes previos, en donde se indica que en el hígado y los riñones se pueden acumular altas cantidades de metales pesados (14). Todas las muestras de roedores se secaron en horno a $105^{\circ} \mathrm{C}$, por espacio de $24 \mathrm{~h}$ de acuerdo a la metodología reportada por (14). Una vez seco, el material biológico se envió al Laboratorio del grupo de contaminación ambiental GDCON de la Sede de Investigaciones Universitarias, SIU, de la Universidad de Antioquia, en donde se determinó la concentración de $\mathrm{Pb}, \mathrm{Cr}, \mathrm{Cd}$ y $\mathrm{Ni}$, por medio de espectroscopia de absorción atómica y siguiendo el procedimiento SW-846 3050B para la determinación de estos metales pesados (15). En resumen, el material biológico se sometió a digestión ácida, mezclando $\mathrm{HNO}_{3}$ y $\mathrm{HClO}_{4}(1: 1)$ a temperatura de ebullición. La mezcla resultante se evaporó con el propósito de reducir el volumen y llevar la muestra a un volumen final de $25 \mathrm{ml}$, en la que se realizó la medición de absorbancia en un espectrómetro GBC 932. El contenido de metales pesados se expresó como $\mathrm{mg} / \mathrm{kg}$ en base a materia seca. Los límites de detección del método empleado (mg/kg) son: $\mathrm{Pb}$ : 0,125, Cr y Ni: 0,250 y Cd 0,050

\section{Resultados}

\section{Muestreo de Artrópodos}

Insectos: La tabla 1 muestra el número de individuos y las familias de los especímenes capturados de acuerdo al tipo de trampa. En total, fueron capturados 534 insectos, distribuidos en 9 órdenes, 51 familias y 75 morfoespecies. El orden más abundante fue Coleoptera con 195 individuos y la familia más abundante fue Formicidae con 122 individuos capturados. Las trampas pitfall con coprocebo mostraron ser las más efectivas para la captura de insectos ya que con ellas se colectaron 195 individuos, mientras que las trampas pitfall con pescado y con fruta en descomposición capturaron 93 y 112 individuos respectivamente. Por medio de la captura con jama se capturaron 134 individuos.

Dentro de los insectos identificados, cabe resaltar la presencia de Palpada vinetorum y Palpada furcata, pertenecientes al grupo conocido como los sírfidos, como primeros registros de estas especies para el Valle de Aburrá. Algunas especies dentro de la familia Syrphidae (una de las más numerosas en el orden Diptera), han sido empleadas en el control biológico de plagas, así como bioindicadores de contaminación ambiental (16).

Arañas: La tabla 2 describe el número de arañas capturadas en el muestreo realizado y la familia a la que pertene- cen. En total se identificaron cinco familias, siendo la Aranaeidae la familia con mayor número de especímenes capturados.

En el muestreo de artrópodos también se identificaron 4 especímenes de la especie Oxidus gracilis (milpiés pertenecientes a la clase Diplopoda: Polydesmida: Strongylosomidae) en las trampas de caída cebadas con pescado y coprocebo.

\section{Muestreo de mamíferos no voladores}

En total se capturaron 10 individuos, todos pertenecientes a la misma especie, Mus musculus Linnaeus, 1758, especie comúnmente conocida como ratón doméstico.

\section{Contenido de metales pesados en tejido animal}

En la tabla 3 se describen las concentraciones de $\mathrm{Pb}, \mathrm{Cr}$, $\mathrm{Cd}$ y Ni encontrados en las muestras de tejido animal capturadas en el Morro de basuras de Moravia. De acuerdo a los resultados observados en la tabla 3 , en los tejidos animales analizados se encontró $\mathrm{Pb}$ y $\mathrm{Cd}$ principalmente.

Los valores máximos de $\mathrm{Pb}$ en tejido animal se presentaron en las muestras de $M$. musculus $(8,05$ y $45,05 \mathrm{mg} / \mathrm{kg}$ en el cuerpo y la cabeza respectivamente). En ninguna de las muestras de artrópodos analizadas se encontraron contenidos detectables de $\mathrm{Pb}$.

En todas las muestras de tejido animal analizado se encontraron contenidos variables de $\mathrm{Cd}$. En el caso de los artrópodos, el contenido varió entre $1,18 \mathrm{mg} / \mathrm{kg}$ (grillos de la familia Acrididae) y 10,31 mg/kg (arañas de la familia Gasteracantha). El contenido de $\mathrm{Cd}$ en las muestras de M. musculus varió entre $0,18 \mathrm{mg} / \mathrm{kg}$ (hígado) y $6,74 \mathrm{mg} / \mathrm{kg}$ (riñón).

De todos los tejidos animales analizados, solo en aquel obtenido de las cucarachas (Blattidae), se encontraron 9,5 $\mathrm{mg} / \mathrm{kg}$ de Ni. En ninguna de las muestras se encontraron concentraciones detectables de $\mathrm{Cr}$.

\section{Discusión}

\section{Diversidad de artrópodos en el cerro de basuras de Moravia}

De acuerdo a los resultados de la caracterización preliminar de la fauna del Morro de Moravia, las condicio- 
Tabla 1. Órdenes y familias de los insectos identificados en el Morro de basuras de Moravia

\begin{tabular}{|c|c|c|c|c|c|}
\hline \multirow[t]{2}{*}{ Orden Familia } & \multicolumn{3}{|c|}{ Método de captura } & \multicolumn{2}{|c|}{ Total capturados } \\
\hline & Jama & $\begin{array}{c}\text { Pitfall } \\
\text { Coprocebo }\end{array}$ & $\begin{array}{l}\text { Pitfall } \\
\text { fruta }\end{array}$ & $\begin{array}{c}\text { Pitfall } \\
\text { pescado }\end{array}$ & \\
\hline Blattodea & & & 5 & 4 & 9 \\
\hline Blattidae & & & 5 & 4 & 9 \\
\hline Coleoptera & 19 & 132 & 34 & 10 & 195 \\
\hline Bostrichidae & & & 2 & & 2 \\
\hline Cantharidae & 2 & & & & 2 \\
\hline Coccinellidae & 5 & & & & 5 \\
\hline Curculionidae & & 1 & & & 1 \\
\hline Chrysomellidae & 7 & 1 & 1 & & 9 \\
\hline Hydrophylidae & & & & 1 & 1 \\
\hline Lagriidae & 3 & & & & 3 \\
\hline Nitidulidae & & 21 & 26 & 5 & 52 \\
\hline Staphylinidae & & 108 & 3 & 3 & 114 \\
\hline Tenebrionidae & 2 & 1 & 2 & 1 & 6 \\
\hline Dermaptera & & 1 & 22 & 3 & 26 \\
\hline Forficulidae & & 1 & 22 & 3 & 26 \\
\hline Diptera & 51 & 36 & 7 & 7 & 101 \\
\hline Agromyzidae & 1 & & & & 1 \\
\hline Callyphoridae & 1 & 1 & 1 & & 3 \\
\hline Chloropidae & 6 & & 1 & 1 & 8 \\
\hline Chyronomidae & 2 & & 3 & & 5 \\
\hline Dolichopodidae & 4 & & & & 4 \\
\hline Drosophilidae & 7 & & 2 & 2 & 11 \\
\hline Lauxaniidae & 5 & & & & 5 \\
\hline Muscidae & 10 & 8 & & 1 & 19 \\
\hline Otitidae & 1 & & & & 1 \\
\hline Phoridae & 1 & & & 2 & 3 \\
\hline Sepsidae & 3 & 5 & & 1 & 9 \\
\hline Sphaeroceridae & & 21 & & & 21 \\
\hline Stratiomydae & & 1 & & & 1 \\
\hline Syllidae & 1 & & & & 1 \\
\hline Syrphidae & 2 & & & & 2 \\
\hline Tachinidae & 2 & & & & 2 \\
\hline Tipulidae & 5 & & & & 5 \\
\hline Hemiptera & 42 & 1 & 5 & 5 & 53 \\
\hline Aphididae & 1 & 1 & & & 2 \\
\hline Cicadellidae & 7 & & 2 & 2 & 11 \\
\hline Coccinellidae & & & 1 & & 1 \\
\hline Coreidae & 9 & & & & 9 \\
\hline Cydnidae & & & 2 & & 2 \\
\hline Lygaeidae & 1 & & & & 1 \\
\hline Membracidae & 3 & & & & 3 \\
\hline Miridae & 17 & & & 2 & 19 \\
\hline Pentathomidae & 2 & & & & 2 \\
\hline Simidae & & & & 1 & 1 \\
\hline Tingidae & 2 & & & & 2 \\
\hline Hymenoptera & 10 & 25 & 38 & 63 & 136 \\
\hline Apidae & 1 & & & & 1 \\
\hline Braconidae & 5 & & & & 5 \\
\hline Chalcididae & 1 & & & & 1 \\
\hline Encyrtidae & 1 & & & & 1 \\
\hline Eupelmidae & & & & 1 & 1 \\
\hline Formicidae & 1 & 25 & 35 & 61 & 122 \\
\hline Ichneumonidae & 1 & & 3 & 1 & 5 \\
\hline Lepidoptera & 5 & & 1 & & 6 \\
\hline Heliconidae & 1 & & & & 1 \\
\hline Noctuidae & & & 1 & & 1 \\
\hline Pieridae & 4 & & & & 4 \\
\hline Orthoptera & 7 & & & 1 & 8 \\
\hline Acrididae & 7 & & & 1 & 8 \\
\hline Total general & 134 & 195 & 112 & 93 & 534 \\
\hline
\end{tabular}


Tabla 2: Número de arañas y familias presentes en el cerro de basuras de Moravia

\begin{tabular}{lc}
\hline Familia & Número de individuos \\
\hline Aranaeidae & 67 \\
Lycosiidae & 12 \\
Salticidae & 4 \\
Thomisidae & 12 \\
Gasteracantha & 14 \\
Total individuos & $\mathbf{1 0 9}$ \\
\hline
\end{tabular}

Tabla 3: Contenido (mg/kg) de $\mathrm{Pb}, \mathrm{Cr}, \mathrm{Cd}$ y Ni en artrópodos y pequeños mamíferos capturados en el cerro de basuras de Moravia

\begin{tabular}{lcccc}
\hline Grupo faunístico y familia & \multicolumn{5}{c}{$\begin{array}{c}\text { Contenido de metales pesados } \\
\text { (mg/kg) }\end{array}$} \\
\hline Artrópodos & Pb & Cr & Cd & Ni \\
Arañas (Gasteracantha) & 5,01 & $<\mathrm{LD} *$ & 10,31 & $<\mathrm{LD}$ \\
Coleópteros (Nitidulidae) & $<\mathrm{LD}$ & $<\mathrm{LD}$ & 2,16 & $<\mathrm{LD}$ \\
Grillos (Acrididae) & $<\mathrm{LD}$ & $<\mathrm{LD}$ & 1,18 & $<\mathrm{LD}$ \\
Cucarachas (Blattidae) & $<\mathrm{LD}$ & $<\mathrm{LD}$ & 3,39 & 9,5 \\
Pequeños mamíferos no voladores & & & \\
Ratón (cabeza, M. musculus) & 45,05 & $<\mathrm{LD}$ & 0,72 & $<\mathrm{LD}$ \\
Ratón (cuerpo, M. musculus) & 8,05 & $<\mathrm{LD}$ & 0,57 & $<\mathrm{LD}$ \\
Ratón (hígado, M. musculus) & $<\mathrm{LD}$ & $<\mathrm{LD}$ & 0,18 & $<\mathrm{LD}$ \\
Ratón (riñón, M. musculus) & $<\mathrm{LD}$ & $<\mathrm{LD}$ & 6,74 & $<\mathrm{LD}$ \\
\hline
\end{tabular}

LD*: Contenido del metal pesado, por debajo del límite de detección

nes actuales del antiguo botadero favorecen el establecimiento de diversidad de familias y especies de insectos y arañas. Una de las principales causas que se ha asociado con la diversidad de artrópodos es la alta disponibilidad de recursos alimenticios. En este sentido, la revegetación espontánea y dirigida por el hombre en lugares rehabilitados, se ha asociado con un incremento en la diversidad de los invertebrados en general, debido a que las plantas colonizadoras proporcionan diversos nichos aptos para su reproducción (17). Nuestro estudio previo (8), mostró un proceso activo y exitoso de revegetación espontánea en el cerro de Moravia, en el cual se encontraron más de 65 especies vegetales pertenecientes a 28 familias botánicas, con predominancia del estrato herbáceo. Este hecho sugiere que el proceso actual de revegetación estimula el establecimiento de artrópodos y otros grupos faunísticos.

De acuerdo a los resultados del presente trabajo, la comunidad de invertebrados identificada en el cerro de Moravia está integrada por órdenes y familias con diversos hábitos alimenticios, desde predadores hasta generalistas. Por ejemplo, las arañas y las cucarachas (Blattidae) son especies omnívoras que pueden establecerse con éxito en diversos ambientes. Dentro del orden Coleoptera, se encuentran familias de fitófagos, (Chrysomellidae), depredadores (Staphylinidae), larvas necrófagas (Callyphoridae) y descomponedores (Nitidulidae). Esta diversidad de hábitos y recursos alimenticios podría contribuir a un proceso de restauración ambiental del cerro. Es de anotar que la diversidad de especies y hábitos encontrados en este muestreo, puede variar ampliamente ya que dependerá de las condiciones propias del cerro de basura, y en especial de posibles procesos de reasentamiento de pobladores del cerro.

De otra parte, en el muestreo realizado no se identificaron especímenes pertenecientes al filo Annelida (lombrices de tierra). Esta observación, si bien no concluyente dadas las características del muestreo realizado en el presente trabajo, puede deberse a condiciones limitantes para el crecimiento de estas especies. En agricultura esto se ha se ha relacionado con prácticas de manejo de alto impacto ambiental negativo (7).

La situación anterior justifica acciones de investigación futura en la cual se establezcan mayor número de estaciones de muestreo así como muestreos repetidos en el tiempo.

\section{Presencia de roedores en el cerro de basuras de Moravia}

Las ratas (Rattus norvegicus y Rattus rattus), y ratones (M. musculus) son los roedores de presencia mayoritaria en botaderos y rellenos sanitarios (7). En el caso del cerro de basuras de Moravia, la presencia de estos roedores está directamente relacionada con la disponibilidad de recursos alimenticios provenientes tanto de la vegetación creciendo sobre la matriz de residuos como de los recursos asociados con la población residente en el Morro de basuras. El muestreo de pequeños mamíferos no voladores solo mostró la presencia del ratón doméstico (M. musculus). Sin embargo, cabe esperar que un muestreo realizado por un periodo de tiempo más extenso, e involucrando mayor número de trampas permita la captura de otras especies de 
pequeños mamíferos comunes a sitios de disposición de residuos.

\section{Contenido de metales pesados}

Los resultados obtenidos en este estudio demuestran que los artrópodos y roedores muestreados contienen en sus tejidos cantidades variables de $\mathrm{Pb}, \mathrm{Cd}$ y Ni. Estos resultados sugieren una transferencia activa de estos contaminantes, desde la matriz de residuos hacia niveles superiores de la cadena trófica. De acuerdo a Rabitsch (18), la ingestión de comida contaminada se presenta como la vía de exposición más frecuente de los invertebrados terrestres a la contaminación por metales pesados. En el caso de los vertebrados, especialmente de los mamíferos, la ruta de exposición más frecuente es el consumo de contaminantes, ya sea por consumo de comida o por ingestión accidental.

Debido al exitoso proceso de revegetación del morro de basuras, es de suponer que las interacciones entre los diferentes niveles tróficos son muy activos en Moravia, y que los diferentes grupos faunísticos están en riesgo de exposición al alto contenido de metales pesados presente tanto en la matriz de residuos como en la vegetación.

\section{Contenido de plomo}

El estudio realizado por (19), señala que algunos especímenes de la especie Apodemos sylvaticus (roedor) capturados en sitios contaminados por la industria del acero y zinc contenían 17,61 y $93,21 \mathrm{mg} / \mathrm{kg}$ de $\mathrm{Pb}$ en sus tejidos de hígado y riñón, respectivamente. De otra parte, el estudio de Rogival et al., (20), reportó información acerca de los contenidos de plomo en tejidos de diversas especies de ratones, capturados en sitios contaminados y no contaminados. En este caso, los sitios no contaminados se asociaron con concentraciones entre 0,5 y $7,0 \mathrm{mg} / \mathrm{kg}$, mientras que los tejidos provenientes de sitios contaminados llegaron hasta $20 \mathrm{mg} / \mathrm{kg}$. El contenido de plomo en el cuerpo de los ratones provenientes de Moravia, superó los $45 \mathrm{mg} / \mathrm{kg}$, sugiriendo la exposición de los ratones en Moravia a altas concentraciones de $\mathrm{Pb}$. En relación a los altos valores encontrados en los especímenes estudiados, es importante mencionar que en nuestro anterior estudio, realizado en el cerro de basuras de Moravia (8), se detectaron contenidos muy altos de MP tanto en muestras de la MR así como en algunas especies vegetales colonizadoras. Las muestras de la matriz de residuos contenían valores tan altos como $9624 \mathrm{mg} / \mathrm{kg}$ de $\mathrm{Pb}, 476,7 \mathrm{mg} / \mathrm{kg}$ de Cr , $13,14 \mathrm{mg} / \mathrm{kg}$ de Cd y $1689 \mathrm{mg} / \mathrm{kg}$ de Ni. En el caso de los tejidos vegetales, se encontraron $123,7 \mathrm{mg} / \mathrm{kg}$ de $\mathrm{Pb}$ en la especie herbácea Lepidium virginicum, 263,7 mg/kg de $\mathrm{Cr}$ en la especie Bidens pilosa y $4,6 \mathrm{mg} / \mathrm{kg}$ de Cd en la especie arbustiva Ricinus communis. Cabe recordar que los límites máximos permitidos para vegetales están por debajo de $1 \mathrm{mg} / \mathrm{kg}$ de metales pesados (21)

Respecto al contenido de plomo en arañas, el estudio de Wilczek et al., (22), reportó contenidos entre 6,3 y $50 \mathrm{mg} /$ $\mathrm{kg}$ de plomo en las especies $X$. nemoralis, A. labyrinthica y $L$. triangularis en dos sitios diferentes de Polonia, con diferentes grado de contaminación por metales. El contenido de $\mathrm{Pb}$ en las arañas capturadas en Moravia se encontró cercano al rango reportado y evidencia la transferencia de este metal a ciertos grupos de artrópodos.

Los resultados del presente estudio permitieron evidenciar el contenido de $\mathrm{Pb}$ en arañas y roedores, por lo que se sugiere que para las condiciones especiales de este lugar, el uso de estos dos grupos faunísticos sea apropiado como indicador de la contaminación por $\mathrm{Pb}$.

\section{Contenido de Cadmio}

En nuestro estudio, el máximo contenido de $\mathrm{Cd}$ se encontró en arañas. Dicho valor $(10,31 \mathrm{mg} / \mathrm{kg})$ está en el rango de aquellos reportados (22), donde se citan contenidos 12 y $102 \mathrm{mg} / \mathrm{kg}$ en las especies $X$. nemoralis, A. labyrinthica y L. triangularis. El estudio realizado por Jelaska et al., (23), reportó contenidos de Cd en diversas especies del género Carabus, colectados en diversos sitios de la región norte de Croacia, contaminados con diferentes metales. En dicho estudio, el contenido de $\mathrm{Cd}$ en coleópteros varió entre 3,5 y 1,79 mg/kg. El contenido de Cd en Coleópteros procedentes de Moravia se determinó en 2,60 mg/kg, valor que excede dicho reporte.

Rabitshc (18), encontró concentraciones de Cd entre 2 y 7 $\mathrm{mg} / \mathrm{kg}$ en varios géneros pertenecientes a la familia Blattidae (cucarachas). En nuestro estudio, las muestras obtenidas de la familia Blattidae contenían 3,39 mg/kg de $\mathrm{Cd}$, valor ubicado dentro del rango del estudio reportado. Los contenidos de metales pesados encontrados en los diferentes estudios indican una alta variabilidad en la absorción de $\mathrm{Cd}$ en los diferentes órdenes de insectos, correspondientes a diferencias propias del ambiente donde se desarrollan las especies, de sus dietas y del grado de exposición de los individuos a los metales pesados.

En el caso del contenido de $\mathrm{Cd}$ en ratones, el estudio reportado por (20), encontró contenidos en tejidos de diversas especies capturadas en sitios contaminados y no 
contaminados. Para este metal, los sitios no contaminados se asociaron con concentraciones entre 0,66 y $3,26 \mathrm{mg} / \mathrm{kg}$, mientras que los tejidos provenientes de sitios contaminados llegaron hasta $20 \mathrm{mg} / \mathrm{kg}$.

La muestra constituida por los riñones de los especímenes de M. musculus obtenidas en Moravia, mostró el mayor contenido de $\mathrm{Cd}(6,74 \mathrm{mg} / \mathrm{kg})$. De acuerdo a la World Health Organization (24), los riñones son los órganos más sensitivos a la exposición de $\mathrm{Cd}$, y en estos órganos se presenta una alta absorción de $\mathrm{Cd}$.

\section{Contenido de Níquel}

De acuerdo a los análisis realizados, se encontraron 9,5 $\mathrm{mg} / \mathrm{kg}$ de $\mathrm{Ni}$ en los tejidos de cucarachas, siendo ésta la única concentración por encima de los límites de detección del método utilizado.

De acuerdo a la determinación del contenido de metales pesados en los diferentes tejidos animales analizados en este estudio, se evidencia una absorción variable de $\mathrm{Pb}$ y $\mathrm{Cd}$, a diferentes niveles de la red alimenticia. Al respecto, la literatura cita que la acumulación de contaminantes en artrópodos y pequeños mamíferos está directamente ligada a la biodisponibilidad de contaminantes, frecuencia de exposición, abundancia de alimentos o recursos contaminados. De forma similar, las interacciones propias en la red alimenticia, afectan la acumulación de contaminantes. De acuerdo a Miller y Clesceri (7) y a Gochfeld (25), la acumulación observada en sitios de disposición de basuras puede modelarse de acuerdo al siguiente esquema: los principales descomponedores, como bacterias y hongos y grupos de pequeños artrópodos como ácaros y protozoos conforman el primer nivel trófico, en el que compiten por materiales orgánicos en descomposición. El grupo de ácaros y pequeños artrópodos son fuente alimenticia de escarabajos, milpiés y moscas, considerados como el segundo nivel trófico. Un grupo paralelo del segundo nivel trófico, se alimenta de tejido vegetal fresco o lignificado, dependiendo de su aparato bucal y preferencia alimenticias. Los individuos pertenecientes a la clase Collembola, que son esencialmente consumidores de hojarasca, son consumidos por los milpiés y otros invertebrados.

Tomando como base los contenidos de Cd encontrados en los tejidos animales procedentes de Moravia, se sugiere que en el antiguo botadero se presenta acumulación de $\mathrm{Cd}$ a través de los niveles de la cadena trófica: herbívoros (grillos) < omnívoros (cucarachas y roedores). Un patrón similar se observó con la acumulación de $\mathrm{Pb}$ : carnívoros inferiores (arañas) < carnívoros superiores (ratón).
Cabe recordar que en términos de investigación toxicológica los roedores han sido considerados como centinelas para la exposición de humanos a metales pesados (26), y que por tanto es posible decir que la población residente en Moravia, estaría también en riesgo de exposición y/o absorción de dichos metales pesados, con consecuencias negativas para su salud.

\section{Conclusiones}

El cerro de basuras de Moravia ofrece recursos alimenticios y nichos suficientes para el establecimiento de varios grupos de artrópodos.

El presente estudio pone de manifiesto una importante alarma en cuanto al posible riesgo de salud al que se enfrentan todos los habitantes de Moravia, debido a la posible transferencia de metales pesados a niveles superiores de la red trófica, los cuales pueden biomagnificarse y acumularse en niveles superiores de la cadena trófica (25).

Se evidenció la absorción de Cd y Pb en niveles de consumidores carnívoros de la cadena trófica (arañas y ratones). Por el contrario, no se encontraron cantidades detectables de Cr y Ni en la mayoría de las muestras analizadas.

Debido a la acumulación de $\mathrm{Cd}$ y $\mathrm{Pb}$ observada en este estudio, se sugiere que la población humana residente también está en riesgo de exposición a la contaminación por metales pesados.

Se requiere de un permanente monitoreo de las poblaciones y su nivel de contaminación por metales pesados, como un indicativo de la evolución de dicha contaminación y el riesgo existente.

Es urgente tomar medidas para el control de la contaminación por metales pesados en Moravia.

De la misma manera, se hace imprescindible el establecimiento de medidas político-administrativas que aseguren el no retorno de población humana al cerro.

\section{Financiación}

Este estudio hizo parte del Macroproyecto "Estudio Piloto para la Recuperación Integral del Morro de Basuras de Moravia”, financiado por El Área Metropolitana del Valle del Aburrá, entidad administrativa y autoridad ambiental de la ciudad de Medellín y la región Metropolitana. 


\section{Conflicto de intereses}

Los autores no presentan conflicto de intereses

\section{Referencias}

1 Villareal H, Álvarez M, Córdoba S, Escobar F, Fagua G, Gast F, Mendoza H, Ospina M, Umaña AM. Manual de métodos para el desarrollo de inventarios de biodiversidad. Programa de Inventarios de Biodiversidad. Instituto de Investigación de Recursos Biológicos Alexander von Humboldt. Bogotá, Colombia. 2006. $236 \mathrm{p}$

2 Ritz K, Black, HIJ, Campbell CD, Harris JA, Word C. Selecting biological indicators for monitoring soils: A framework for balancing scientific and technical opinion to assist policy development. Ecological Indicators 2009; 9: 1212-1221

3 Talmage SS, Walton BT. Small mammals as monitors of environmental contaminants. Reviews of Environmental Contamination and Toxicology 1991; 119: 47-145.

4 Alloway BJ. Heavy Metals in Soils, second ed. Blackie Academic \& Professional, London.1995, 368 p.

5 Vrijheid M. Health effects of residence near hazardous waste landfill sites: a review of epidemiologic literature. Environmental Health Perspectives 2000; 108 (1): 101-112

6 Kabata-Pendias, A. Soil-plant transfer of trace elements-an environmental issue. Geoderma 2007; 122: $143-9$

7 Miller PA, Clesceri NL (Eds.). Waste sites as biological reactors: characterization and modeling. Lewis Publisher, Boca Ratón, FL. 2003, 398 p.

8 Sánchez MS, Barahona R, Salazar C, Arroyave C, Bedoya A. Informe final del grupo de flora, fauna y fitorremediación. En: Estudio piloto para la recuperación del Morro de Moravia, Fase I. Informe final de Proyecto. Escuela de Ingeniería, Facultad de Minas, Universidad Nacional de Colombia. 2009

9 ICONTEC, Instituto Colombiano de Normas Técnicas. Norma técnica colombiana 5167. Productos para la industria agrícola. Productos orgánicos usados como abonos o fertilizantes y enmiendas de suelo. Bogotá, Colombia. 2004

10 ASCP Guidelines. Quality Criteria for Composts and Digestates from Biodegradable Waste Management,
Schoenbuehl, Switzerland. 2001. http://www.vksasic.ch/acrobatreader/vks_richtlinie_english.pdf. (Consultado en Enero de 2009)

11 Underwood EC, Fisher B. The role of ants in conservation monitoring: If, when, and how. Biological Conservation 2006; 132: 166-182

12 Turnbull AL. Ecology of the true spiders (Araneomorphae). Annual Review of Entomology 1973; 18: 305348

13 Drickamer LC, Springer LM. Methodological aspects of the interval trapping method with comments on nocturnal activity patterns in house mice living in outdoor enclosures. Behavioural Processes 1998; 43 : $171-181$

14 Sánchez-Chardi A, Peñaroja A, Olivera C, Nadal J. Bioaccumulation of metals and effects of a landfill in small mammals. Part II. The wood mouse, Apodemus sylvaticus. Chemosphere 2007; 70: 101-109

15 EPA. "Method 3050B (SW-846): Acid Digestion of Sediments, Sludges, and Soils," Revision 2. 1996. http://www.epa.gov/sam/pdfs/EPA-3050b.pdf (Consultado en Enero de 2009)

16 Sommaggio D. Syrphidae: can they be used as environmental bioindicators? Agriculture, Ecosystems and Environment 1999; 74: 343-356

17 Grimbache PS, Catterall CP. "How much do site age, habitat structure and spatial isolation influence the restoration of rainforest beetle species assemblages?" Biological Conservation 2007; 135(1): 107-118

18 Rabitsch WB. Metal accumulation in arthropods near a lead/zinc smelter in Arnoldstein, Austria. Environmental Pollution 1994; 90, 221- 237

19 Damek-Poprawa M, Sawicka-Kapusta MK. Damage to the liver, kidney, and testis with reference to burden of heavy metals in yellow-necked mice from areas around steelworks and zinc smelters in Poland. Toxicology 2003; 186(1- 2): 1-10.

20 Rogival D, Scheirs J, Blust R. Transfer and accumulation of metals in a soil dietewood mouse food chain along a metal pollution gradient. Environmental Pollution 2007; 145: 516-528

21 Codex Alimentarius Commission, Commission Regulation (EC) No 1881/2006, Official Journal of the European Union, Available from the website http:// eur-lex.europa.eu/LexUriServ/site/en/oj/2006/1_364/ 1_36420061220en00050024.pdf, 2006. 
22 Wilczek G, Babczynska A, Augustyniak M, Migula $\mathrm{P}$. Relations between metals ( $\mathrm{Zn}, \mathrm{Pb}, \mathrm{Cd}$ and $\mathrm{Cu}$ ) and glutathione-dependent detoxifying enzymes in spiders from a heavy metal pollution gradient. Environmental Pollution 2004; 132: 453-461

23 Jelaska L, Blanusa S, Jelaska MS. Heavy metal concentrations in ground beetles, leaf litter, and soil of a forest ecosystem. Ecotoxicology and Environmental Safety 2007; 66: 74-81

24 World Health Organization (WHO). Cadmium: Air Quality Guidelines, second Ed. World Health Organi- zation, Regional Office for Europe, Copenhagen, Denmark. 2000

25 Gochfeld M. Cases of mercury exposure, bioavailability, and absorption. Ecotoxicology and Environmental Safety 2003; 56: 174-179

26 Shore RF, Rattner BA. (Eds.). Ecotoxicology of Wild Mammals. Wiley, Chichester, New York, Weinheim 2001, 736 p. 